\section{Creación de escenarios convergentes entre docencia, investigación y extensión: la Residencia Integral del Profesorado en Comunicación}

\section{Paula Contino}

Docente y Secretaria de Extensión y Vinculación de la Facultad de Ciencia Política y Relaciones Internacionales de la Universidad Nacional de Rosario, Argentina

continopaula@yahoo.com

\author{
Mariela Daneri \\ Docente y Sub - Secretaria de \\ Extensión y Vinculación de la Facultad \\ de Ciencia Política y Relaciones \\ Internacionales de la Universidad \\ Nacional de Rosario, Argentina. \\ mares@arnet.com.ar
}

Integración de la docencia y la extensión /

Desafíos de gestión

RECEPCIÓN: 21/06/16

ACEPTACIÓN FINAL: 03/09/16

\section{Resumen}

Este artículo acerca la mirada a los procesos contemporáneos que rigen el pulso de las tres funciones sustantivas de la universidad: docencia, investigación y extensión. Podría decirse que esta última, fruto de la jerarquización de años recientes, ha desarrollado progresivamente una capacidad creativa y creadora trayendo consigo nuevos desafíos para pensar la arquitectura institucional universitaria en clave relacional, es decir, un lugar donde converjan de manera estratégica funciones, actores, saberes y disciplinas. Sobre estas ideas paradigmáticas se levanta la Residencia Integral del Profesorado en Comunicación, creado recientemente en la Facultad de Ciencia Política y Relaciones Internacionales de la Universidad Nacional de Rosario, constituyendo así un espacio de enseñanza -aprendizaje de carácter integral y dialogante entre teoría, práctica, investigación y socialización de las tramas emergentes.

Palabras clave

- Integralidad

- Funciones

- Dialógico

- Institucionalidad

- Conocimiento

\section{Resumo}

Este artigo aproxima o olhar sobre os processos contemporâneos que regem o pulso das três funções essenciais da universidade: docência, pesquisa e extensão. Pode-se dizer que esta última, produto da hierarquização dos últimos anos, desenvolveu uma capacidade criativa trazendo consigo novos desafios para pensar a arquitetura institucional universitária na chave relacional, quer dizer, um lugar onde convergem estrategicamente funções, atores, saberes e disciplinas. Sobre estas ideias paradigmáticas se constrói o Estágio Integral do Professorado em Comunicação, criado recentemente na Faculdade de Ciência Política e Relações Internacionais da Universidade Nacional de Rosario, constituindo dessa forma um espaço de ensino-aprendizagem integral e que debate com a teoria, a prática, a pesquisa e a socialização das redes emergentes.

Palavras-chave

- Integralidade

- Funções

- Dialógico

- Instituicionalidade

- Conhecimento
Para citación de este artículo

Contino, P. y Daneri, M. (2016). Creación de escenarios convergentes entre docencia, investigación y extensión: la Residencia Integral del Profesorado en Comunicación. En Revista +E versión digital, (6), pp. 130-137. Santa Fe, Argentina: Ediciones UNL. 


\section{Breves ideas preliminares sobre la institucionalidad universitaria \\ "no hay práctica docente sin curiosidad, sin incompletud, \\ sin capacidad de intervenir en la realidad, \\ sin capacidad de ser hacedores de la historia siendo, \\ a su vez, hechos por la historia." Paulo Freire (2006)}

La universidad es una forma particular de organizar la institución educativa y esa organización se edifica sobre la política que la concibe y la estrategia que le da sustento. En un juego paradojal, la educadora Graciela Frigerio nos revela que:

"las instituciones trabajan haciendo que lo objetivable pase a formar parte del mundo interno. $Y$ haciendo que el mundo interno pueda expresarse en cuestiones objetivables (...) las instituciones (permiten) que lo está afuera pase a estar adentro de (nuestra) cabeza. Incluso los psicoanalistas (sostienen) que la identidad no es algo que un sujeto singular pueda hacer solo. La identidad siempre es social y política". (2004:8)

En este marco, cabe destacar que entendemos a las universidades como:

"instituciones sociales, o sea formaciones socio-culturales que han sido producidas por los sujetos y sus prácticas (...) pasando a constituir elementos determinantes $y$ estructurantes de las mismas. Lo que se hace, cómo se hace y los fundamentos por los cuales se hace, toman carácter de instituido y pasan a ordenar, a pautar. A partir de aquí la institución y sus normativas (...) aparecen marcando las prácticas de los sujetos. Se constituyen así las organizaciones, sede material que en el tiempo y espacio concretos son las productoras de la legalidad normativa, a la vez que surge el espacio de las confrontaciones, búsquedas y conflictos generadores de lo nuevo". (Peralta, 2012:2)

Esto nos permite comprender lo instituido como aquello que está determinado, como el conjunto de normas y valores dominantes, como ley estructurante de lo humano, de lo social.

Sin embargo, lejos de la pretensión conservadora que se funda en el criterio de la persistencia ambiental, para entender el carácter dinámico de las instituciones es necesario reconocer, también, la presencia de una fuerza instituyente, nacida como cuestionamiento, disputa de sentido o negación de la norma instaurada.

Dentro de esta lógica y acercando la mirada a los procesos contemporáneos que marcan el pulso de las tres funciones sustantivas de la universidad: docencia, investigación y extensión, podría decirse que esta última, fruto de la jerarquización de años recientes, ha desarrollado progresivamente una capacidad creativa y creadora trayendo consigo nuevos desafíos para pensar la arquitectura institucional universitaria en clave relacional, es decir, un lugar donde converjan de manera estratégica funciones, actores, saberes y disciplinas.

Sobre estas ideas paradigmáticas se levanta la Residencia Integral del Profesorado en Comunicación, creado recientemente en la Facultad de Ciencia Política y Relaciones Internacionales de la Universidad Nacional de Rosario.

\section{Aprendizajes en contextos relacionantes}

"El proceso educativo nos impone la necesidad de inventar situaciones creadoras de saberes." Paulo Freire (2006)

Para comenzar cabe señalar que la extensión, en tanto materialización de la función social universitaria, ha cobrado en los últimos tiempos espesura y resonancia en los debates de la educación superior. Los primeros años del siglo XXI, tanto en Argentina como en los países de la región, se revigorizó el rol del Estado y por consiguiente el concepto y la práctica de lo público, en tanto espacio inherente a lo político, impactando de diferentes modos en la vida académica. Esto ha colocado a la extensión en una posición saludable en relación a décadas pasadas, recobrando para sí, la idea de la construcción del conocimiento en diálogo interactuante con diversos sujetos colectivos, como así también la visión de una sociedad más justa e incluyente en el marco del desarrollo humano.

La extensión en el debate contemporáneo intenta alejarse de conceptos difusionistas, transferenciales o salvíficos de épocas atrás, ubicándose en el espacio de la interacción y la alteridad. Concepto filosófico que remite a la capacidad y al reconocimiento de ser otro en una multiplicidad de visiones, es decir, tener el otro en nosotros; pensando ( $y$ anhelando) una comunidad integrada que no niegue la diferencia. En ese sentido, Graciela Frigerio (2006:4) recupera el planteo de Jaques Rancière, quien asegura que "para que haya sociedad, es necesario, que todo sujeto sienta que forma parte de esa sociedad, y para que esto ocurra necesita tener su parte", es decir, esa sociedad tiene que poder reconocer y distribuir bienes materiales, culturales y simbólicos para generar sentimiento de pertenencia. Cuando una sociedad no distribuye genera desigualdades y asimetrías que deja a diversos sectores sin presente, y por lo tanto, sin futuro.

En este sentido, advertimos una revalorización de la sociedad por parte de la universidad, que se expresa en múltiples programas, proyectos e intervenciones territoriales (aunque esto no ocurre de modo homogéneo en las instituciones de la educación superior), no sólo porque ésta la financia a través de los recursos del Estado nacional, sino porque le ha dado un "sentido" particular a su práctica. Actualmente esta tarea cobra "agenciamiento" en el debate universitario, al tiempo que encuentra diversas formas de institucionalización en los procesos de formación y gestión. 


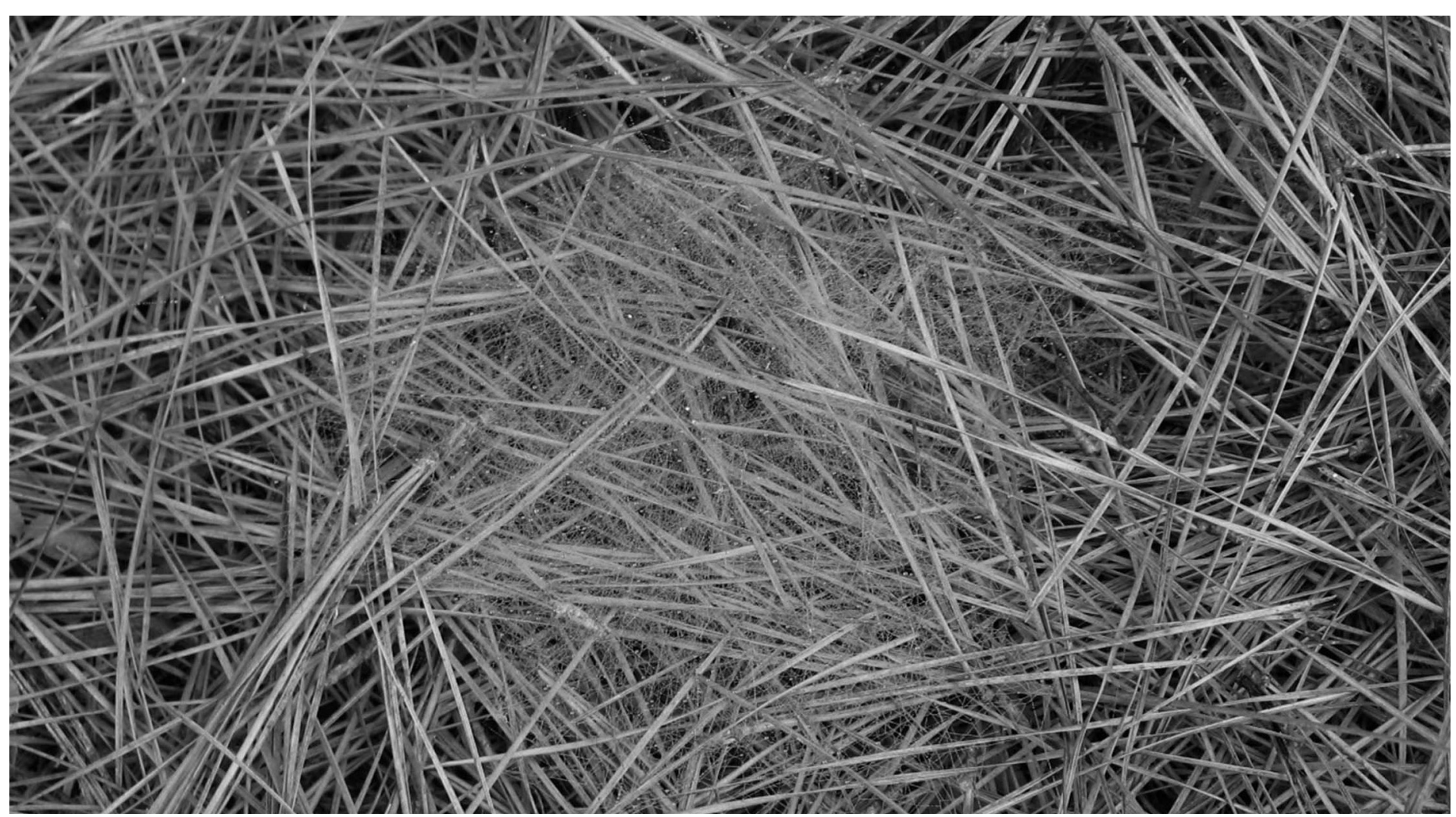

(c) Cecilia lucci

\section{6}

la extensión en el debate contemporáneo intenta

alejarse de conceptos

difusionistas, transferenciales

o salvíficos de épocas atrás, ubicándose en el espacio de la interacción y la alteridad 
La noción deleuziana de agenciamiento (Deleuze, 2010) emerge aquí como un complejo de líneas que pone en relación ideas, cuerpos y enunciados. Develar su entramado o promover nuevas urdes presupone visibilizar modos de hablar, de relacionarnos, de accionar y de pensar. Este acontecimiento ha permitido que la cuestión de su jerarquización, haya adquirido fuerza significante en determinados espacios de la vida académica logrando su inclusión a través de mecanismos de gestión, técnicos-administrativos; en algunos casos cátedras específicas (tal es el caso que narraremos más adelante); y prácticas socio-comunitarias; o programas, proyectos y voluntariados.

De todos modos, tal como se ha señalado con anterioridad, frente la riqueza de los debates y los avances alcanzados en este campo, creemos importante resaltar que esta discusión no debe focalizarse, ni estancarse en la extensión en sí misma, en tanto forma reduccionista, para no transformase en aquello que critica: la perspectiva unívoca que deviene de saberes y funciones compartimentadas o fragmentadas. No olvidemos que nuestra vida institucional se organiza y se denomina en "claustros" universitarios. Entonces, el eje de la interrogación debería orientarse hacia discusiones sustanciales que pongan en el foco de escena cómo avanzar hacia una universidad integrada de manera inteligible y sensible de cara a la complejidad de los procesos actuales. Si bien este debate no es nuevo, no por ello ha perdido su vigor, máxime aún, a dos años de cumplirse el centenario de la Reforma Universitaria de Córdoba que amplió de manera incomparable el derecho a la educación superior.

En el marco de esta lógica que proyecta su horizonte en la interrelación dialogante de la docencia, la investigación y la extensión, encontramos un camino posible para el diseño de una nueva Residencia Integral para el Profesorado en Comunicación, que transita su joven existencia desde hace un año y medio. Cabe señalar que esta carrera en función de su objeto y según los lineamientos ministeriales se encuadra en los estudios de "interés público". Por lo tanto, y de manera coherente con lo expuesto, esta definición influyó en su proyección, y entendió al espacio público como lugar de relación y de identificación que pone en contacto la vida urbana y con la expresión comunitaria.

\section{Los desafíos que inspiraron la trama de la Residencia Integral}

Los integrantes de la Secretaría de Extensión y Vinculación de la Facultad de Ciencia Política y Relaciones Internacionales que formamos parte del Comité Académico de Elaboración del Profesorado, a la hora de bosquejar este trayecto partimos de ciertas preguntas que fueron guiando el trabajo:

¿Cuál es el criterio de pertinencia que nos orienta? ¿Con qué actores y de qué modos realizamos las prácticas socioeducativas mediante la articulación inteligente de las funciones a fin de construir saberes vinculados con las necesidades del entorno?

¿Cómo establecemos filiaciones societales desde esta mirada democrática del proceso de enseñanza aprendizaje?

Estos fueron algunos de los interrogantes que acompañaron la planificación de la Residencia Integral del Profesorado y con los que nos encontramos cada vez que pensamos en una institución territorializada, es decir, una institución en contexto. Pero antes de continuar, es menester señalar qué entendemos por "territorio" en tanto concepto teórico y metodológico que, según nuestra perspectiva, describe y analiza el juego de las relaciones sociales y relaciones de fuerza que se establecen entre los sujetos en los diversos campus en términos de Bourdieu.

El territorio, desde una noción polifónica, apela en su complejidad a diferentes aspectos como: multidimensionalidad, multiculturalidad, singularidad y multirreferencialidad. De este modo, el territorio es comprendido como geografía en tensión, como el mapeo de la disputa, el consenso, la reproducción y la resistencia. Otra vez, nos situamos aquí en el "entre" de los instituidos y los instituyentes que marcan la vida y la historia de las organizaciones.

\section{Aulas sociales para la innovación curricular}

En este nuevo siglo las formas y los lugares donde se produce conocimiento son múltiples y complejos, de ahí que los currículos y los modos de gestión político-académica deben ser cada vez más abiertos y flexibles. En este contexto se vuelve necesaria la innovación, en tanto novedad que interroga las certidumbres, provoca la curiosidad y va en búsqueda de una creatividad desafiante. Las miradas disciplinares deben dar paso a visiones más amplias. El perímetro de un cerco disciplinar no nos alcanza para intervenir; tenemos que salir de la comodidad de los paradigmas que nos hacen sentir seguros a fin de subvertir las respuestas dadas, suspender las certezas y animarnos al desafío de preguntas irreverentes. Nuestros saberes son insuficientes, por si solos no dan cuenta de la contingencia, de los equilibrios inestables y de las interdependencias que construyen nuestro mundo. Las actividades científicas y académicas tienen que romper los mosaicos feudalizados en la producción del conocimiento. En definitiva, sigue siendo esencial sostener "el principio de una impertinencia epistémica del conocimiento (en tanto) condición de su libertad y su fecundidad críticas" (Naishtat, 2003:3).

Porque cuando hablamos de conocimiento académico, éste solo adquiere potencial y relevancia cuando se articula necesariamente con los saberes sociales, populares e históricos en una jerarquía polivalente. No sin dificultad, entendemos que las respuestas no son únicas y definitivas, que en muchos casos son provisoras, y esto nos exige la búsqueda de los intersticios y los mestizajes culturales. Más aun, cuando estamos pensando en futuros profesionales del campo educativo. 


\section{La contextura del Profesorado en Comunicación}

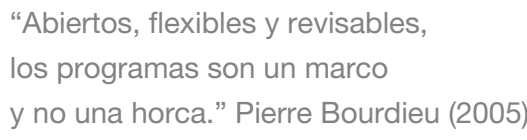

"Abiertos, flexibles y revisables,

los programas son un marco

y no una horca." Pierre Bourdieu (2005)

El Profesorado de Comunicación Educativa tiene como objetivo formar docentes capaces de producir, organizar y cocrear conocimientos y acciones específicas referidas a la educación en comunicación. Conceptualizando ésta última como una dimensión transversal a los procesos de diversa índole que articulan la vida de cada sujeto y la vida societal que, en esta era digitalizada y globalizada, no cesa de generar innovaciones en materia de tecnologías de la información y la comunicación (TIC) en el marco del lenguaje digital. Modificando de manera continua los esquemas de percepción, pensamiento y comportamiento humanos, transformando así, los modos de interrelación cultural tanto en los procesos de subjetivación como los de socialización.

En este marco, se entiende que la relación de la educación superior con las dinámicas contextuales de lo local y lo global, requieren de una transformación de la tarea educativa de un nuevo profesor en educomunicación desde una perspectiva integral que cuestione la producción, distribución y circulación del conocimiento, y construya la docencia, extensión e investigación como diálogo interactuante entre los diversos sujetos sociales, históricos y académicos. En consecuencia, el conocimiento tendría que borrar ciertas parcelaciones para dejar de ser:

"divisible en sus componentes de origen, (ya que) no se acomoda con la dinámica particular de las disciplinas, sino con la dinámica de los problemas (educativos) complejos, en el marco de una noción de ciencia posnormal, afectada simultáneamente por un elevado nivel de incertidumbre y por una alta cuota de negociación y deliberación entre actores heterogéneos." (Naishtat, 2003:11)

En este contexto se genera un:

"sistema distribuido en el que la universidad pierde el monopolio de la producción de conocimiento y debe asociarse (necesariamente) a otros actores institucionales. A la homogeneidad de las especializaciones (y funciones) sucede ahora una amplia heterogeneidad que debe hallar sin embargo coherencia centrípeta en la dinámica organizacional”. (Naishtat, 2003: 11)

La síntesis de esta nueva mirada nos aleja de viejos arquetipos y nos propone la figura del "trabajador de la educación" con una agenda marcada por problemáticas, y no por las agendas abstractas de las disciplinas tradicionales del ámbito educativo específico. Sin dudas, en este contexto aparece un sentido más profundo para la idea de la "pertinencia" entre lo que realizan las instituciones de la educación superior y lo que la sociedad espera realmente de ellas.
En este proceso, las tradiciones teóricas de la enseñanza más elitista van perdiendo su centralidad, mientras el mundo académico desde su mirada actual, impulsado por el panorama de una "educación integral", se vuelve más sensible y receptivo a las problemáticas de su tiempo, particularmente al sujeto mediado y mediatizado.

De este modo, la Residencia Integral se propone como espacio abierto, dialogante y en permanente problematización, es decir, inscripta en el campo de lo político, que posibilita la idea de un nuevo orden basado en la construcción de nuevos sentidos, que navega sobre la concepción del llamado conocimiento socialmente significativo.

\section{Toda acción educativa es, indefectiblemente, una acción comunicativa}

En líneas generales, el diseño curricular del Profesorado de Comunicación Educativa, cuyo dictado implica un acuerdo de gestión entre la Facultad de Ciencia Política y Relaciones Internacionales y la Facultad de Humanidades y Artes de la Universidad Nacional de Rosario, se organiza en torno a la interrogación crítica de tres cuestiones fundamentales que articulan los dominios usuales de la comunicación y la educación:

- Los procesos de comunicación como producto de relaciones materiales y simbólicas y la disputa cultural, histórica y social por el sentido. Los escenarios socioculturales contemporáneos signados por la globalidad y la fragmentación.

- Las instituciones educativas frente a nuevas subjetividades pedagógicas y mediáticas: reconfiguraciones comunicativas del saber y del narrar. Nuevos lenguajes y nuevas alfabetizaciones en la cultura audiovisual-digital.

- Las políticas culturales y comunicacionales en el campo de la educación. Las TIC como entorno, dispositivo y lenguaje en las prácticas educativas.

La Residencia del Profesorado de Comunicación Educativa está pensada como un conjunto articulado y desplegado en el transcurso de dos niveles, que encuentran su ubicación en el tercer y cuarto año de la carrera, con características propias en cada uno de ellos que se expresan en los Contenidos Mínimos; reservando la especificidad de la tradicional Residencia Docente para el último año, mientras la Residencia Integral se ubica en el tercer año y constituye un espacio distintivo destinado a una práctica educativa tendiente a reconocer y desarrollar capacidades estratégicas para el trabajo docente cooperativo en diferentes contextos, tanto escolares como sociocomunitarios.

Asimismo, se plantea como el ámbito de convergencia de un conjunto de procesos que promueven el diálogo permanente entre teoría, práctica, investigación y socialización de las construcciones conceptuales emergentes. 


\section{6}

la relación de la educación superior

con las dinámicas contextuales

de lo local y lo global, requieren

de una transformación de la tarea

educativa de un nuevo profesor en

educomunicación

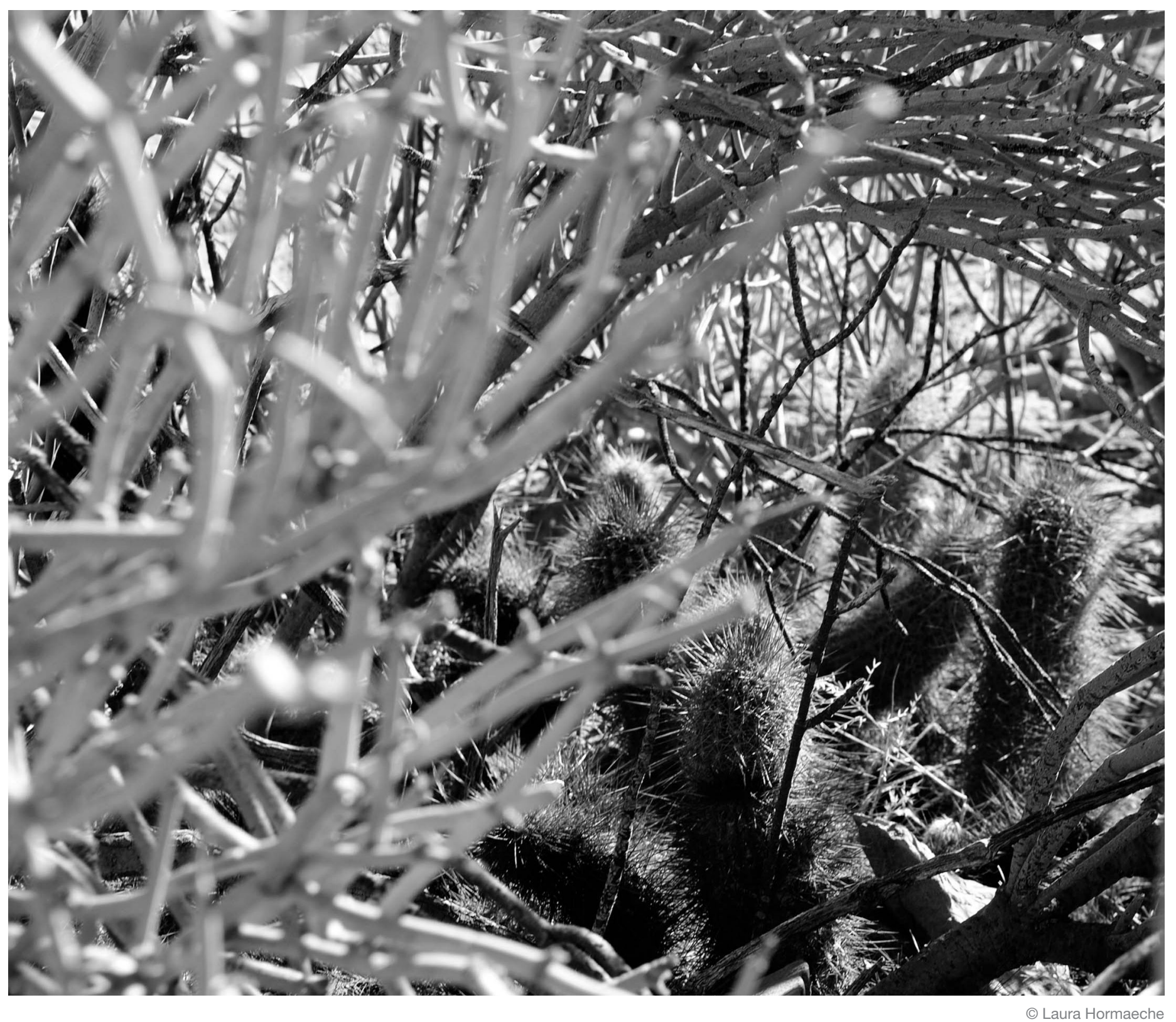


Este enfoque se inscribe en una búsqueda epistemológica y pedagógica y en el desafío de integrar las experiencias territoriales con los procesos de formación y producción de conocimiento, en un intento creativo que resignifique las prácticas de enseñanzaaprendizaje, y por lo tanto las formas de hacer, investigar y rehacer las tradiciones pedagógicas vinculadas a los saberes comunicacionales, tanto académicos como populares en ecosistemas culturales hipertecnológicos.

\section{Las singularidades de Residencia Integral}

Como síntesis del planteo anterior, la Residencia Integral del Profesorado en Comunicación Educativa se construye a través de Trayectos Curriculares Integradores (TCl) configurados en tres niveles que, tal como se anticipó, encontrarán su lugar en el tercer año de la malla curricular, con una cátedra compuesta para tales fines con una carga horaria de 120 horas. A continuación describimos su fisonomía y las temáticas que darán cuerpo a los contenidos programáticos.

Cabe insistir que los "Trayectos Curriculares Integradores" imaginaron la formación de un profesional distinto, comprometido con su medio, y se curriculariza docencia, extensión e investigación enmarcada en el concepto de integralidad señalado.

En el primer nivel ( $\mathrm{TCl} 1$ ), la modalidad de trabajo se desarrolla en el aula, se pone el énfasis en una primera aproximación al desarrollo y conceptualización de la extensión universitaria, los modelos educativos que la contienen, el reconocimiento del proceso de enseñanza-aprendizaje en la tríada ciudadanía, compromiso y participación, las dimensiones de la solidaridad y el reconocimiento de la alteridad cultural como proceso cognoscente y constitutivo de la integración.

Desde un enfoque propio de las ciencias sociales, se ponen en juego las discusiones en torno a los pares dicotómicos: objetividad/ subjetividad, neutralidad/implicancia, racionalidad/corporalidad, entre otros; las construcciones de sentido común y el sentido crítico, y se contrapone la concepción positivista de la ciencia con las teorías dialécticas a través de casos vinculados a problemáticas locales y regionales que posibiliten el debate y la reflexión situada. El segundo nivel de trabajo ( $\mathrm{TCl} 2$ ) interviene en diferentes territorios educativos, tanto del ámbito formal como "no formal" (poniendo en discusión el concepto que refiere a lo "no formal o informal") con las herramientas teóricas metodológicas aprendidas. Se procura que la producción del conocimiento amplíe sus horizontes a través de la investigación-acción-participación, utilizando, por ejemplo, el diagnóstico participativo y sus posibles líneas de intervención en la organización educativa. También: "el mapeo colectivo forma parte de este trayecto, en tanto herramienta lúdica y creativa que facilita la construcción de un relato colectivo sobre un territorio. La información obtenida se socializa en un espacio horizontal de encuentro que apunta a elaborar saberes y condensarlos en un soporte común (el mapa). Está pensado como una instancia de construcción (compartida) y participación abierta, permitiendo el conocimiento crítico de diversas realidades a partir de la memoria cotidiana y los saberes no especializados. Hacer mapas sirve para agilizar el trabajo y la reflexión (...) a la hora de pensar nuestro territorio. Un mapa nos brinda la posibilidad de tomar distancia, de imaginar un vuelo de pájaro que nos facilite descifrar las conexiones entre las diversas problemáticas a fin de cuestionarlas y elaborar alternativas de resistencia, organización y cambio. La cartografía es un proceso en permanente mutación, un punto de partida disponible a ser retomado por otros, una plataforma desde la cual idear otras actividades". (Iconoclasistas, 2013:4, 5 y 6)

El tercer trayecto ( $\mathrm{TCl} 3$ ) aspira a que los estudiantes desarrollen capacidades para construir una planificación teórico-práctica sobre ejes transversales de la enseñanza, de modo que el conocimiento no aparezca fragmentado o discurra en forma dispersa y pierda su potencia y vitalidad. La idea central es generar las condiciones de posibilidad para "suscitar el aprendizaje de manera integrada". A manera de ejemplo, y en términos generales, podemos decir que no serán ya la Revolución de Mayo o la Revolución Francesa por sí mismas un tema de estudio o un hecho puntual sino los movimientos revolucionarios que marcaron el pulso epocal los que formarán parte de los nuevos currículos.

Para alcanzar tales objetivos se proponen diversos espacios de articulación territorial en el ámbito educativo formal e "informal" y recrear la idea del nomadismo o del migrante que funda la lógica del encuentro, que sale a la búsqueda y se deja permear por los interrogantes en cada uno de los recorridos que siga el estudiante. En consecuencia, las intervenciones territoriales, mediante convenios específicos, se materializan en distintos espacios, a saber:

1) Organizaciones de la Sociedad Civil o colectivos vinculados a diferentes temáticas como:

- Derechos humanos.

- Ambiente: promoción y preservación.

- Salud: prevención y promoción.

- Educación en contextos de encierro.

- Comunicación para usuarios de salud mental. 


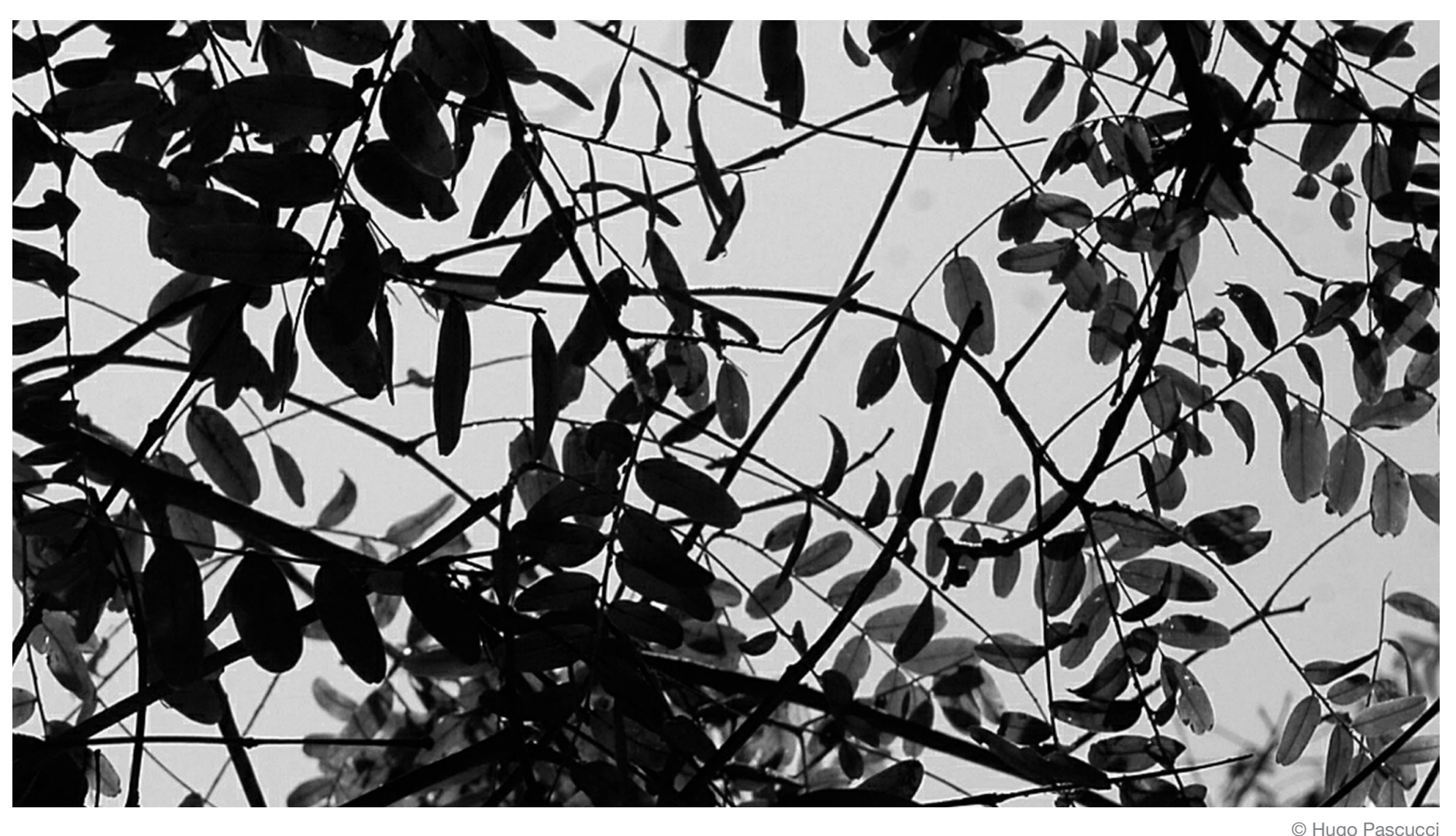

2) Bibliotecas populares.

3) Cooperativas.

4) Fundaciones mixtas.

5) Escuelas primarias y medias.

6) Institutos terciarios, no universitarios y universitarios.

7) Medios públicos y comunitarios.

8) Instituciones públicas del estado multinivel vinculadas al ámbito educativo y a las políticas públicas.

Tal como fue mencionado, el Profesorado en Comunicación Educativa está cursando su segundo año y el próximo será el momento de poner en acto la Residencia Integral. Sin lugar a dudas, la implementación de este espacio curricular interpelará la reciprocidad de nuestra institución educativa con la sociedad que da sentido a su práctica. La perspectiva contemporánea e instituyente de la integralidad encontrará una nueva razón para su devenir, al tiempo que el suceso educativo tendrá el desafío de participar en el espacio público como esfera de realización de los derechos ciudadanos.
Referencias bibliográficas

Bourdieu, P. (2005). Capital cultural, Escuela y espacio Social (6ºd.). Buenos Aires: Siglo XXI Editores.

Deleuze, G. y Guattari, F. (2010). Mil mesetas. Madrid: Pre-textos. Freire, P. (2006). El grito manso. Buenos Aires: Siglo XXI Editores.

Frigerio, G. (2004). Institución, Conceptos y Perspectivas. Exposición de la Dra. Graciela Frigerio. Seminario de formación en el marco del curso de formación gremial, viernes 22 de octubre. Asociación Trabajadores del Estado (ATE-CDP). Santa Fe, Argentina. Recuperado el 12/05/2016 de: http://www.amsafe.org.ar/ formacion/images/2013-cursodirectores/Eje6/Graciela\%20Frigerio\%20-\%20Institucion\%20conceptos\%20y\%20perspectivas.pdf

Iconoclasistas (2013). Manual de mapeo colectivo. Recuperado el 12/05/2016 de: https://issuu.com/iconoclasistas/docs/manual_de_mapeo_2013?e=0/7840606 Naishtat, F. (2003). Universidad y conocimiento: por un ethos de la impertinencia epistémica. Espacios de Crítica y producción, 30. Buenos Aires.

Peralta, M.I. (1987). Recontrucción de prácticas extensionistas en la UNC; contextos históricos y teóricosideológicos. Reflexiones en ocasión de los 400 años de la UNC". Revista EXT: 2012. Cita de Dubost; J. y Levy, A. El análisis Social en Guattari, F. y otros, La intervención institucional. México. 